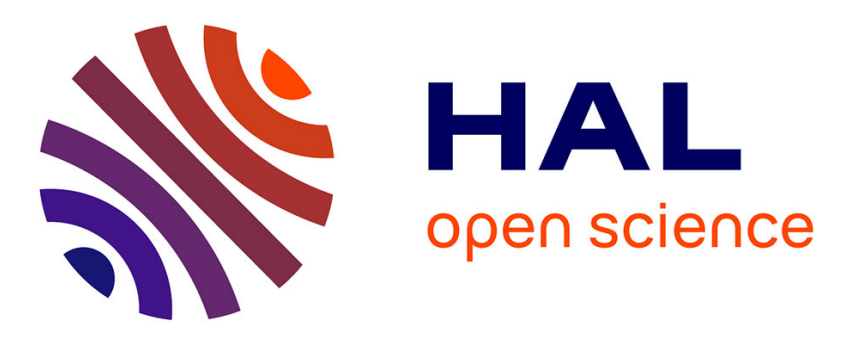

\title{
Ionic mobility in DNA films studied by dielectric spectroscopy
}

Abdelkader Kahouli, Jessica Valle Orero, Jean-Luc Garden, Michel Peyrard

\section{To cite this version:}

Abdelkader Kahouli, Jessica Valle Orero, Jean-Luc Garden, Michel Peyrard. Ionic mobility in DNA films studied by dielectric spectroscopy. European Physical Journal E: Soft matter and biological physics, 2014, 37 (9), pp.82. 10.1140/epje/i2014-14082-5 . ensl-01069225

\section{HAL Id: ensl-01069225}

\section{https://hal-ens-lyon.archives-ouvertes.fr/ensl-01069225}

Submitted on 29 Sep 2014

HAL is a multi-disciplinary open access archive for the deposit and dissemination of scientific research documents, whether they are published or not. The documents may come from teaching and research institutions in France or abroad, or from public or private research centers.
L'archive ouverte pluridisciplinaire HAL, est destinée au dépôt et à la diffusion de documents scientifiques de niveau recherche, publiés ou non, émanant des établissements d'enseignement et de recherche français ou étrangers, des laboratoires publics ou privés. 


\title{
lonic mobility in DNA films studied by dielectric spectroscopy
}

\author{
Abdelkader Kahouli ${ }^{1}$, Jessica Valle-Orero ${ }^{2}$, Jean-Luc Garden ${ }^{3,4}$, and Michel Peyrard ${ }^{5}$ \\ 1 Grenoble Electrical Engineering Laboratory (G2ELab), Université Joseph Fourier, CNRS, 25 rue des Martyrs, BP 166 , 38042 \\ Grenoble cedex 9, France. \\ 2 Institut Laue Langevin, BP 156, 6, rue Jules Horowitz 38042 Grenoble Cedex 9, France. \\ 3 CNRS, Institut NEEL, F-38042 Grenoble, France \\ 4 Université Grenoble Alpes, Institut NEEL, F-38000 Grenoble, France \\ ${ }^{5}$ Laboratoire de Physique, Ecole Normale Supérieure de Lyon, 46 allée d'Italie, 69364 Lyon Cedex 07, France.
}

September 29, 2014

\begin{abstract}
Double-helix DNA molecules can be found under different conformational structures driven by ionic and hydration surroundings. Usually, only the B-form of DNA, which is the only form stable in aqueous solution, can be studied by dielectric measurements. Here, the dielectric responses of DNA molecules in the A- and B-form, oriented co-linearly within fibres assembled in a film have been analyzed. The dielectric dispersion, permittivity and dissipation factor, have been measured as a function of frequency, strength voltage, time, temperature and nature of the counter-ions. Besides a high electrode polarization component, two relaxation peaks have been observed and fitted by two Cole-Cole relaxation terms. In the frequency range that we investigated $\left(0.1 \mathrm{~Hz}\right.$ to $\left.510^{6} \mathrm{~Hz}\right)$ the dielectric properties are dominated by the mobility and diffusivity of the counter-ions and their interactions with the DNA molecules, which can therefore be characterized for the A- and B-forms of DNA.
\end{abstract}

PACS. 87.14.gk DNA - 77:22.-d Dielectric properties of solids and liquids

\section{Introduction}

Studies of the dielectric dispersion of DNA have a long history as they started even before its molecular structure had been discovered [1] and were regularly pursued since then [2-9]. There is a double motivation for these studies. First they provide data on DNA properties. For instance the earlier studies intended to determine its molecular weight [1] or dipole moment. Second DNA is a highly charged polymer due to the charged phosphate groups along the strands. As its solutions also contain positive counter-ions that stabilize its molecular structure, it is a perfect model polyelectrolyte. This is what motivated investigations by pioneers of the dielectric theory, such as Mandel [10] or Cole [11]. Then speculations on the mechanism of electronic conduction along DNA were at the origin of various attemps to measure its electrical conductivity, including studies of wet-spun solid samples [12]. This long history does not mean that all questions are solved, as discussed for instance in a more recent paper [9].

There are technical difficulties in the measurements because DNA samples are highly conducting due to the presence of counter-ions. This leads to very strong electrode polarization effects, which makes the detection and analysis of the other dielectric relaxations harder. But there are also many difficulties in the understanding of the results. Dielectric spectroscopy is sensible to all sources of polarization fluctuations. In the DNA solutions, used most of the experiments up to now, this includes the internal polarization of the DNA molecules, the dynamics of the counter-ions, but also the motion of the DNA molecules themselves that contributes to a large orientational polarization.

The present study re-examines the dielectric properties of DNA from a completely new perspective because, instead of studying DNA solutions, we investigate films made of DNA fibers, i.e. a sample in a solid phase, in which the molecules are oriented. This has multiple interests. First it eliminates the orientational contribution of the polarization. Second, as we know the orientation of the molecules with respect to the electric field used in the measurements, we can take advantage of it in the assignment of the observed relaxations, which is often a difficult question in dielectric spectroscopy. But there is a third interest which is perhaps even more important, we can study the different molecular forms of DNA [13]. The familiar double helix schematically drawn in the famous Watson-Crick paper [14], with its backbone made of sugar-phosphate strands connected by flat molecular groups, the AT and GC base pairs, which are perpendicular to the helix axis, is the B-form of DNA. This is the structure which is present in biological cells and in DNA aqueous solutions. DNA can however adopt other molec- 
ular structures, particularly the A-form, which is also a double helix but has its base pairs inclined with respect to the axis and positioned in such a way that the diameter of the helix is significantly larger than in the B-form. The A-form of DNA is the structure from which Rosalind Franklin, who was studying fiber samples, obtained the nicest X-ray diagrams of DNA because its fibers are more crystalline than those of the B-form. It seems that it is actually the X-ray pattern of the A-form which gave Watson and Crick the clue for the B-DNA structure [15].

The A-form is obtained when the hydration of DNA is reduced. This is why it cannot be observed in aqueous solutions. Working with fiber samples we study here two types of DNA films, a highly hydrated film of Li-DNA (i.e. with $\mathrm{Li}^{+}$counter-ions) in which DNA is in the Bform, and a weakly hydrated Na-DNA film (i.e. with $\mathrm{Na}^{+}$ counter-ions) in which DNA is in the A-form [16]. This allows us to perform what we believe is the first dielectric study of A-DNA.

Our measurements are performed in the low-frequency range $\left(0.1 \mathrm{~Hz}\right.$ to $\left.510^{6} \mathrm{~Hz}\right)$ which is suitable to study the properties resulting from the presence of the counterions, which play a large role in the DNA structure and stabilization because they screen the repulsion between the charged phosphates in the strands. In the presence of $\mathrm{Li}^{+}$ions DNA remains in the B-form in a wider watercontent [17]. This is why we use Li-DNA to study DNA in its B-form and Na-DNA to investigate the A-form.

\section{Experimental Methods}

\subsection{Sample Preparation}

The samples were prepared using the "wet spinning technique" $[18,19]$. A solution of DNA, extracted from salmon testes (Sigma), in the presence of a counter-ion, is spinned to form DNA fibers. In the process, the solution is injected through a spinneret, which pre-orients the molecules, into an alcoholic solution, which flows down a glass tube about 1 meter long. The flow completes the orientations of the molecules, and the alcoholic medium leads to their aggregation into a long fiber which is then rolled over a glass cylinder, still in alcoholic solution. The fibers tend to aggregate to each other and form a thin film made of oriented DNA molecules. Fig 1 shows the optical observation of the fiber structure of a DNA film. Measurements using gel diffusion show that the DNA molecules in a film have lengths of 20000 base pairs or larger, i.e. of about $6 \mu \mathrm{m}$ or larger [20].

The structure of DNA is strongly dependent on the ionicity of its surrounding medium. DNA strands include negatively charged phosphate groups so that, in the double helix their repulsion tends to destabilize the helix. This is compensated by counter ions, which screen the phosphate repulsive forces. When DNA is heated to high temperature (typically 85 to $100^{\circ} \mathrm{C}$, depending on its sequence and the ionicity of the solution) the thermal fluctuations can nevertheless break the double helix structure and separate the two strands. This is known as the "melting" of DNA.
The melting temperature is higher in solutions with a high ionic content. When DNA melting occurs in a film, this destabilizes the organized film structure. The denaturated DNA strands are highly flexible and their entropic elasticity leads to a collapse of the fiber and film structure. When they are cooled down again after the thermal denaturation of DNA, the films exhibit a glassy behavior associated to a complex organization of the DNA polymeric strands [21].

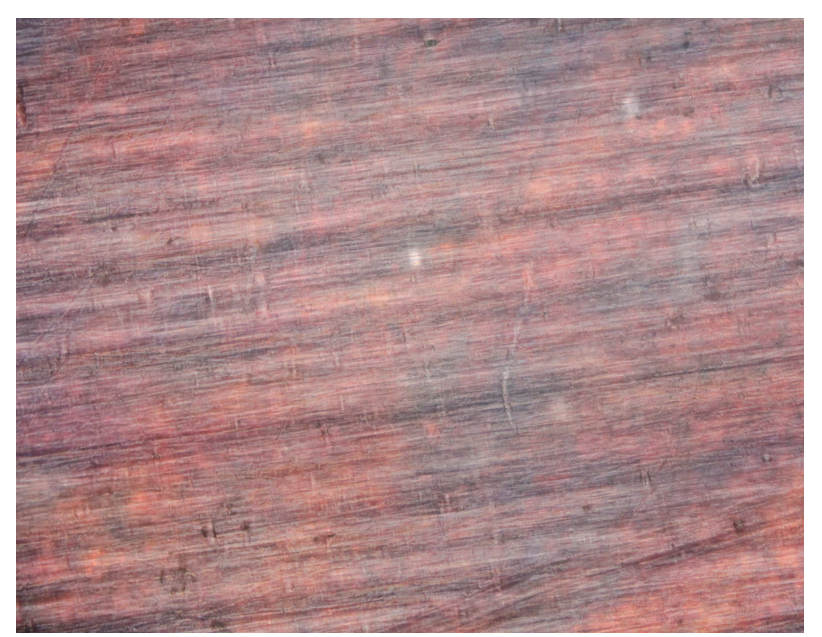

Fig. 1. Optic Microscopy image of a film of Li-DNA, where the orientation of the fibers is visible.

DNA films were obtained from two different solutions. Li-DNA is prepared from a solution with $0.6 \mathrm{~g} / \mathrm{L}$ of DNA, i.e. about $10^{-3} \mathrm{M} / \mathrm{L}$ of base pairs, and a high concentration of lithium chloride $\mathrm{LiCl} 0.4 \mathrm{M}$. Therefore there are about $400 \mathrm{Li}^{+}$ions per base pair in the solution. Na-DNA is obtained with a solution containing $1.58 \mathrm{~g} / \mathrm{L}$ of DNA $\left(\approx 2.610^{-3} \mathrm{M}\right.$ for base pairs) and $0.15 \mathrm{M}$ sodium chloride $\mathrm{NaCl}$, correspondig to about $57 \mathrm{Na}^{+}$ions per base pair. However the ionic concentration in the films is certainly different from that in the injected solution and it is not exactly known.

Once the films are made they are humidified at room temperature under different relative humidities $(\mathrm{RH})$, in deionized water, in order to induce the desired DNA conformation. For the purpose of our experiments, the films containing sodium $(0.15 \mathrm{M})$ were humidified at $56 \% \mathrm{RH}$ and those containing lithium $(0.4 \mathrm{M})$ at $92 \% \mathrm{RH}$. This preparation process leads to two different DNA structures [16]. The highly hydrated Li-DNA is in B-form while the weakly hydrated Na-DNA is in A-form.

The films used in the dielectric measurements were of the order of $80 \mu \mathrm{m}$ thick, and weighted about $50 \mathrm{mg}$. As the DNA molecules lie in the film plane, their are orthogonal to the electric field.

\subsection{Dielectric Experiments}

Dielectric measurements were performed with a Novocontrol Alpha-A high resolution Spectrometer. The complex 
impedance $Z^{*}$ was measured in the frequency range $0.1 \mathrm{~Hz}$ to $510^{6} \mathrm{~Hz}$ at various temperatures from room temperature to $80^{\circ} \mathrm{C}$ or $90^{\circ} \mathrm{C}$. Some datasets were however restricted to $10^{6} \mathrm{~Hz}$ when the conductivity of the samples became to high to allow reliable measurements. From the impedance measurements and the geometry of the measuring cell one can derive the complex dielectric response function versus frequency $\epsilon^{\star}(f)=\epsilon^{\prime}(f)-i \epsilon^{\prime \prime}(f)$, or the conductivity $\sigma(f)$ of the sample.

The sample cell uses gold plated electrodes. The lower electrode is a $40 \mathrm{~mm}$-diameter disk, heated from below, supporting the $\approx 80 \mu \mathrm{m}$-thick DNA film, which is pressed under an upper disk, having a diameter of $20 \mathrm{~mm}$, surrounded by a guard ring. To measure the dielectric properties of DNA fibers within a controlled relative humidity, the measurements were carried out in a hydrothermal chamber (Votsch "VC7018" industrie technick Fisher Biolock Scientific) which can maintain relative humidities (RH) from 0 to $100 \% \mathrm{RH}$.

We tested the possible influence of an applied voltage on the dielectric response of the system to make sure that the measurements were carried in the linear response regime and to check that the electrical stress due to the applied potential did not lead to changes in the sample (change in the conformation and configuration of the molecular chains, a possible electrolysis of water, or even an electrical denaturation of the double helix). Voltages between 0.001 to $3 \mathrm{~V}$ were applied on test samples. These preliminary studies showed that there is a threshold voltage of $\sim 1 \mathrm{~V}$ above which the dielectric response enters a non-linear regime. The dielectric constant $\epsilon^{\prime}$ and, in particular, the dissipation factor $\tan \delta$ showed an irreversible response after the sample was subjected to a voltage higher than this threshold voltage. All the data reported in this work were obtained with an applied voltage of $0.1 \mathrm{~V}, \approx 1250 \mathrm{~V} / \mathrm{m}$ taking into account the tickness of our samples, low enough to stay in the linear regime.

Even for low applied voltage, dielectric measurements on complex samples such as biological samples [22] show some time evolution before reaching a steady state. This phenomenon was also observed in the DNA films investigated in the present study. It is likely to be caused by some reorganization of the ionic distribution in the presence of an electric field. A waiting time of 12 to 16 minutes was necessary to allow the dissipation factor $\tan \delta$ to stabilize before recording data.

\section{Experimental results}

\subsection{Li-DNA}

Figure 2 shows the variation of the real and imaginary parts, $\epsilon^{\prime}(f)$ and $\epsilon^{\prime \prime}(f)$, of the dielectric response function of the Li-DNA film versus frequency in logarithmic scales at different temperatures. Their very high values, particularly at low frequency, and quasi-linear variation in the log-log plots suggests that the dielectric response of the
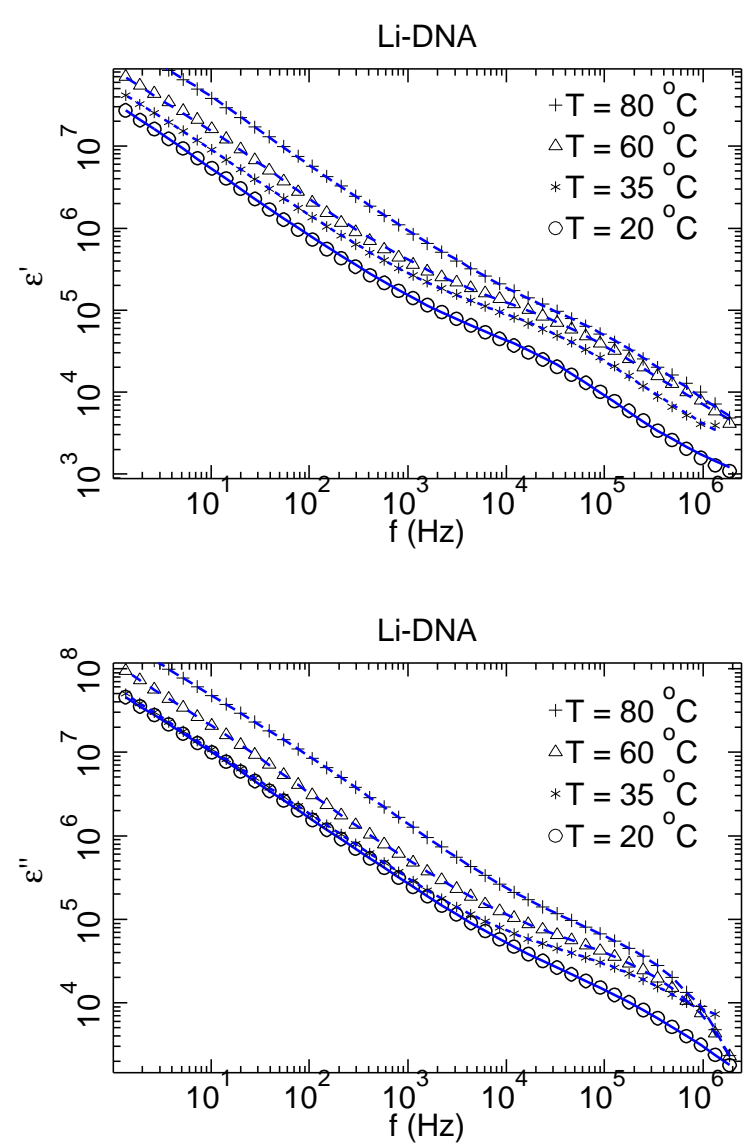

Fig. 2. Variation of the real(top panel) and imaginary (bottom panel) parts of the dielectric response function $\epsilon^{\star}(f)$ of the Li-DNA film versus frequency at different temperatures. The points are the measurements and the lines show the theoretical fits discussed in 3.2 .

sample includes the contribution of a strong electrode polarization effect associated to mobile charge carriers within the sample. This is confirmed by the Cole-Cole plot of the impedance shown in Fig. 3 and the variation of the conductivity $\sigma$ shown in Fig. 4

The Cole-Cole plot of the impedance shows the presence of a least two types of dielectric responses. The arc near the origin corresponds to high frequencies and it can be associated to the relaxation that appears as a deviation to the straight line in the log-log plot of the dielectric response function (Fig. 2). The conductivity $\sigma=2 \pi f \epsilon_{0} \epsilon^{\prime \prime}$, where $\epsilon_{0}$ is the dielectric permittivity of vacuum, plotted in Fig. 4, shows a rather large conductivity contribution which approximately follows a power law with however two deviations from a perfect power law. In the high frequency range, around $10^{5} \mathrm{~Hz}$, one notices a large deviation from the straight line in the log-log plot, associated to the relaxation also visible on Fig. 2 showing the imaginary part of the response function. Although it appears weaker on the plot of the conductivity, one also notices a low frequency relaxation, around $10 \mathrm{~Hz}$ at $T=20^{\circ} \mathrm{C}$. 


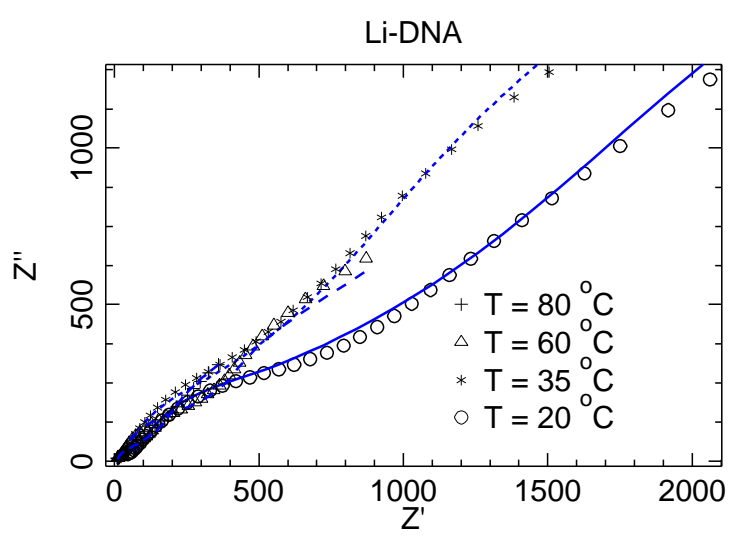

Fig. 3. Cole-Cole plot of the complex impedance $Z^{\star}$ of the LiDNA film at different temperatures. The points are the measurements and the lines show the theoretical fits discussed in Sec. 3.2.

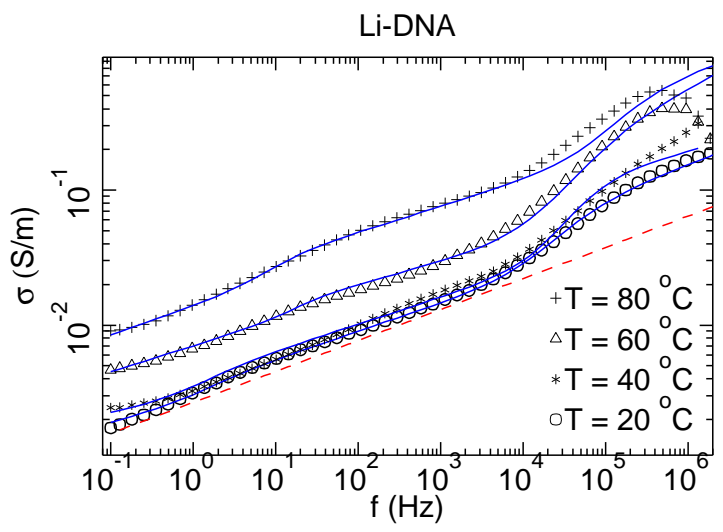

Fig. 4. Variation of the conductivity $\sigma(f)$ of the Li-DNA film versus frequency at different temperatures. The points are the measurements and the full (blue) lines show the theoretical fits discussed in Sec. 3.2. The (red) dash line is a guide to the eye to illustrate the deviation from a simple power law at $T=20^{\circ} \mathrm{C}$.

\subsection{Analysis of the dielectric spectra.}

As shown by the shape of the dielectric response function and the Cole-Cole plot of the impedance, the conductivity of the sample is responsible for a strong electrode polarization effect. For our experiments, as the voltage is sufficiently low to exclude ionic reactions at the electrodes, there is no static conductivity component. The polarization effect, which leads to a space- and time-dependent local ionic concentration, can therefore be described by a power law dependence on the frequency $f[23,24]$ as observed from the conductivity plot. Moreover, as discussed above, the experimental results on the Li-DNA film point out the presence of two dielectric relaxations. To analyze the data quantitatively we have therefore fitted the re- sponse function with the following expression

$$
\begin{aligned}
\epsilon^{\star}= & \frac{A}{f^{\delta}}-i \frac{B}{f^{\delta}}+\epsilon_{\infty}+ \\
& \frac{\Delta \epsilon_{1}}{1+\left(i f / f_{1}\right)^{\beta 1}}+\frac{\Delta \epsilon_{2}}{1+\left(i f / f_{2}\right)^{\beta 2}}
\end{aligned}
$$

We have chosen to describe the relaxations by Cole-Cole expressions [25], each one depending on three parameters, its frequency $f_{i}$, the prefactor $\Delta \epsilon_{i}$ measuring the strength of the relaxation, and the exponent $\beta_{i} \leq 1$ that indicates how this relaxations deviates from a simple Debye relaxation. The more general model introduced by Havriliak and Negami [26], which contains an additional parameter, could give a better fit, however, owing to the large polarization component that dominates the dielectric spectrum and makes the fit of the relaxation part harder, the reliability of the parameter determination would be limited if we introduced too many parameters. This is why we used the simpler Cole-Cole expression, which is sufficient to fit the data with a rather good accuracy as shown in figures 2, 3 and 4 which superimpose the theoretical curves obtained from Eq. (1) to the experimental points.

For this multi-parameter problem, in order to get reliable fits we proceed in steps. The parameters of the electrode polarization component, $A, B, \delta$, are estimated first, from a fit of the conductivity in the low frequency range. Then the conductivity is fitted in the high frequency range to determine parameters of the strongest relaxation, and then in the low frequency range for the second relaxation, before performing a global optimization of the curves $\epsilon^{\prime}(f), \epsilon^{\prime \prime}(f)$. Figure 5 showing the loss tangent $\tan \delta=\epsilon^{\prime \prime} / \epsilon^{\prime}$ versus frequency gives an idea of the quality of the fits that can be obtained with Eq. (1). When examining this figure which shows that the fit is not perfect, one should however keep in mind that $\tan \delta$, of the order of unity, is the ratio of two quantities which are in the range $10^{3}$ to $10^{8}$, which are fitted with parameters which are not adjusted independently for the numerator and denominator.

Figure 6 shows the variation versus temperature of the parameters used to fit the dielectric response of the LiDNA film versus temperature. Before discussing these results in Sec. 4 let us first present the experimental results obtained on a Na-DNA film.

\subsection{Na-DNA}

As shown by Fig. 7 the variation of the dielectric permittivity of the Na-DNA-film is qualitatively similar to that of the Li-DNA film, although the measured values are approximately three orders of magnitude smaller. This is due to the lower conductivity of the Na-DNA-film (Fig. 8) related to its lower ionic content. As a result the relaxation part of the dielectric spectrum is more apparent. Figure 9 , showing the loss tangent, still points out to the existence of two dielectric relaxations within the frequency domain that we investigated. Therefore the analysis of the data has been performed with the same model function, Eq. (1). The results are shown on Fig. 10. 


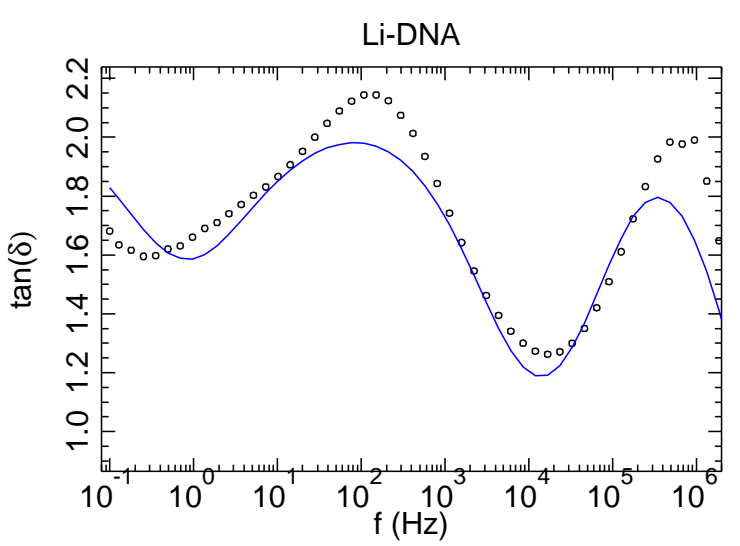

Fig. 5. Loss tangent $\tan \delta=\epsilon^{\prime \prime} / \epsilon^{\prime}$ versus frequency for the Li-DNA film at $T=20^{\circ} \mathrm{C}$. The points are the measurements and the line shows the theoretical fit with Eq. (1).

\section{Discussion}

Although there are quantitative difference between the results obtained on Li-DNA and Na-DNA their global similarity is remarkable if one thinks that the structures of the DNA molecules are different in the two films. The common feature of the samples is that they are made of fibers of aggregated molecules, which are highly charged polymers due to the phosphate groups in DNA strands, with lengths of a few microns, and have a high ionic content, larger for Li-DNA than Na-DNA. The dielectric measurements show that these global characteristics are more important than the precise molecular structure to determine the dielectric properties of the films on the range of $0.1 \mathrm{~Hz}$ to $510^{6} \mathrm{~Hz}$.

In both cases, the dielectric results are dominated by a strong electrode polarization effect. Such phenomena are complex, but, in the case of a solid electrolyte they can be related to the conductivity of the sample as well as the diffusivity of the charge carriers [27,28]. A theory was proposed for the case of a single type of charge carriers [28]. Although the structure of DNA films is more complex than the case of solid electrolytes dicussed in [28] there is some similarity because the system has both fixed charges (the phosphate groups) and mobile carriers (the $\mathrm{Li}^{+}$or $\mathrm{Na}^{+}$counter ions). Electrode polarization effects are generally considered as a nuisance for dielectric measurements [27]. Indeed in our case they tend to mask other dielectric relaxation effects. Nevertheless, if we cannot get rid of them because they are intrisically linked to the properties of the sample, we can extract some physical information from their analysis because they provide indirect information on the carrier mobility and diffusivity. In such an approach it is important to realize that the first two terms of Eq. (1) are not a feature of the sample alone because they also depends on the geometry of the measurement cell. However their magnitude and frequency dependence, i.e. the time-dependent charge relaxation, is deeply connected to the mobility and diffusivity of the charge carriers [27] in the bulk. This appears clearly in the theoretical (a)

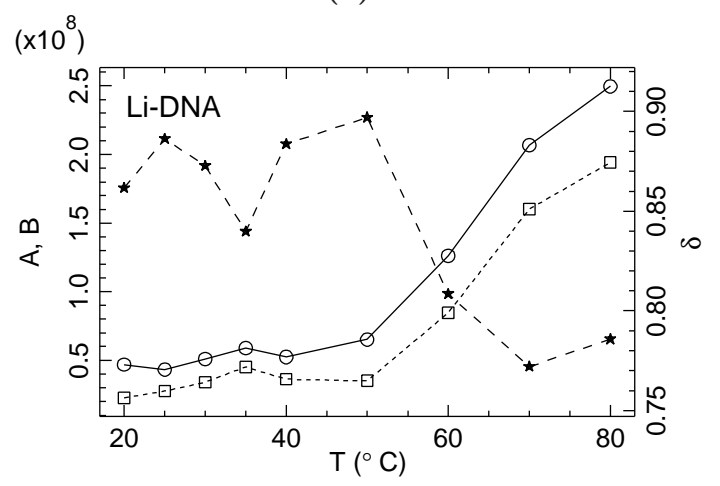

(b)

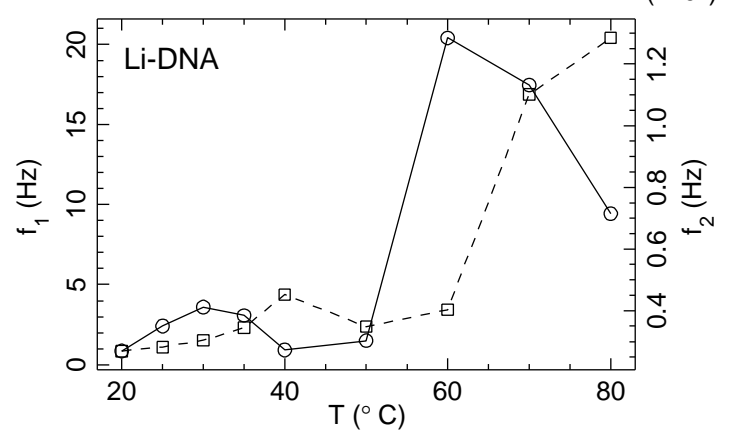

(c)

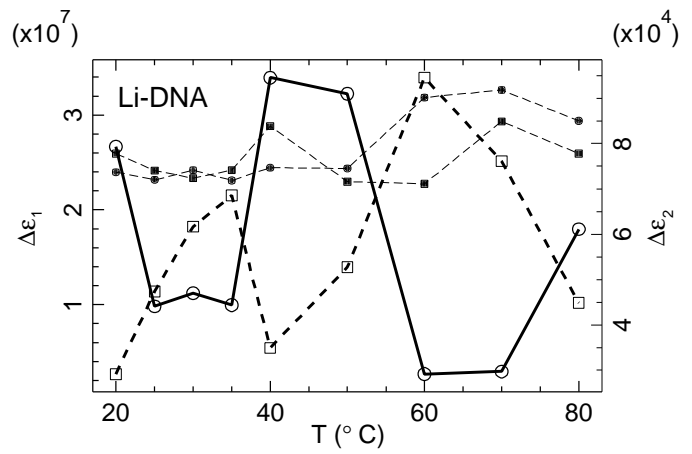

Fig. 6. Variations versus temperature of the parameters of the model fitting the dielectric response of the Li-DNA film: a) parameters $A$ (open circles), $B$ (open squares), $\delta$ (stars) of the polarization component. b) Frequencies $f_{1}$ (open circles), $f_{2}$ (open squares) of the two relaxations. c) Strengths $\Delta \epsilon_{1}$ (open circles) and $\Delta \epsilon_{2}$ (open squares) of these relaxations. The variation of the Cole-Cole exponents $\beta_{1}$ (closed circles) and $\beta_{2}$ (closed squares) are also shown on a scale ranging from 0 to 1 .

analysis [28] of the electrode polarization effects because these two properties are the basic ingredients entering in the fundamental equations of the theory (Sect. 2 of [28]).

The measurements (Figs 4 and 8 ) show that the conductivity of the Li-DNA film is about $10^{4}$ times larger than the conductivity of the Na-DNA film. The ionic concentration per DNA base pair is 7 times larger in the solution used to preapare the Li-DNA film than in the solution 

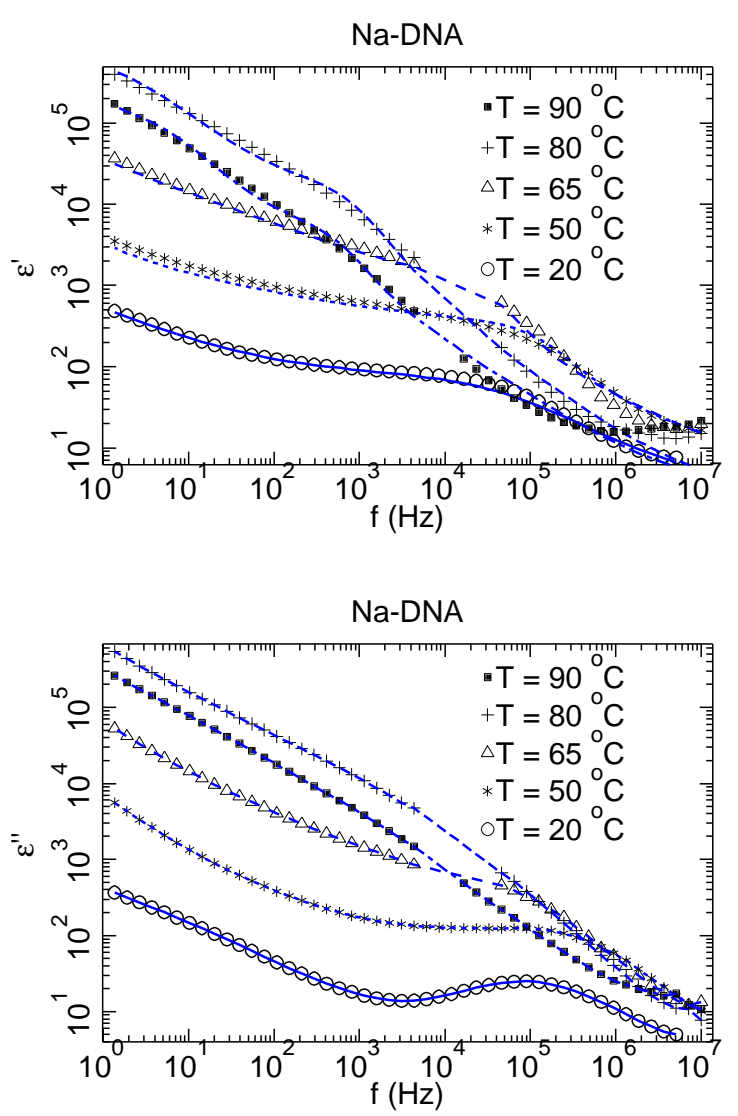

Fig. 7. Variation of the real(top panel) and imaginary (bottom panel) parts of the dielectric response function $\epsilon^{\star}(f)$ of the Na-DNA film versus frequency at different temperatures. The points are the measurements and the lines show the theoretical fits discussed in 3.2 .

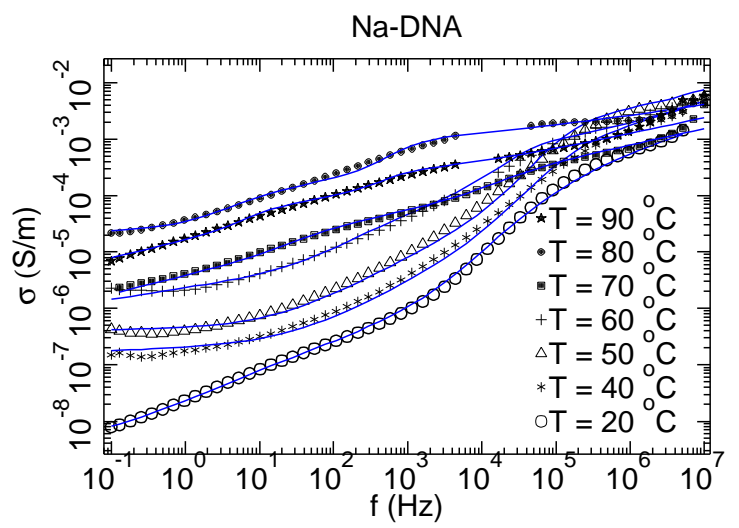

Fig. 8. Variation of the conductivity $\sigma(f)$ of the Na-DNA film versus frequency at different temperatures. The points are the measurements and the full (blue) lines show the theoretical fits discussed in Sec. 3.2.

used to prepare the Na-DNA film. It is likely that the ratio of the ionic concentrations in the films themselves is of the same order of magnitude. Therefore the charge carrier

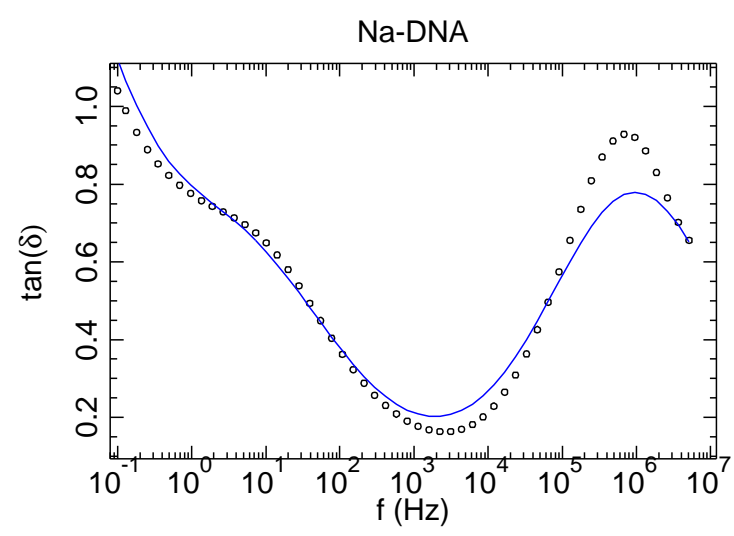

Fig. 9. Loss tangent $\tan \delta=\epsilon^{\prime \prime} / \epsilon^{\prime}$ versus frequency for the Na-DNA film at $T=20^{\circ} \mathrm{C}$. The points are the measurements and the line shows the theoretical fit with Eq. (1).

density is not the only explanation of the much higher conductivity of the Li-DNA film. The mobility of the carriers must also be significantly larger in the Li-DNA film. In both samples the coefficients $A$ and $B$ used to model the electrode polarization in Eq. 1 increase sharply with temperature. Figure 11 shows an Arrhenius plot of $\ln (A)$ and $\ln (B)$ versus $1 / T$, where $T$ is the absolute temperature. It indicates that their variation is dominated by thermal activation, although there are additional effects that contribute to its variation. A linear fit gives an activation energy of about $0.31 \mathrm{eV}$ for the Li-DNA film and $1.16 \mathrm{eV}$ for the Na-DNA film. These results are crude because the temperature range of our study is limited, and there are significant deviations from a perfect Arhhenius law due to additional phenomena. However the large temperature variation which is observed for the coefficients $A$ and $B$ suggests an activation process and, even if the values of the activation energies have to be taken with caution, the difference observed between the two films is certainly significant. The larger value of the activation energy and the lower carrier mobility in the Na-DNA film, in which the molecules are in the A-form, is consistent with what is known about the structures of DNA fibers. The fibers of the B-form, which are more hydrated, leave more room for the motion of the counter-ions, whereas the fibers of the A-form have a higher crystalline content in which the molecules are closely packed [17]. The average volume per base pair is approximately two times larger in Li-DNA at $92 \% \mathrm{RH}$ than in $\mathrm{Na}-\mathrm{DNA}$ at $56 \% \mathrm{RH}$ and therefore it is not surprising to find a significantly higher barrier for ionic motion in the Na-DNA film, and a lower charge carrier mobility.

Moreover, for the Na-DNA film, the results show a significant drop of the conductivity at high temperature. This is visible on Fig. 8 because the curve at $90^{\circ} \mathrm{C}$ is below the curve at $80^{\circ} \mathrm{C}$, and this is reflected in the drop of the parameters $A$ and $B$ at the highest temperature in Fig. 10a. This can be related to the thermal denaturation of the DNA molecules, which is observed in the Na-DNA film 
(a)

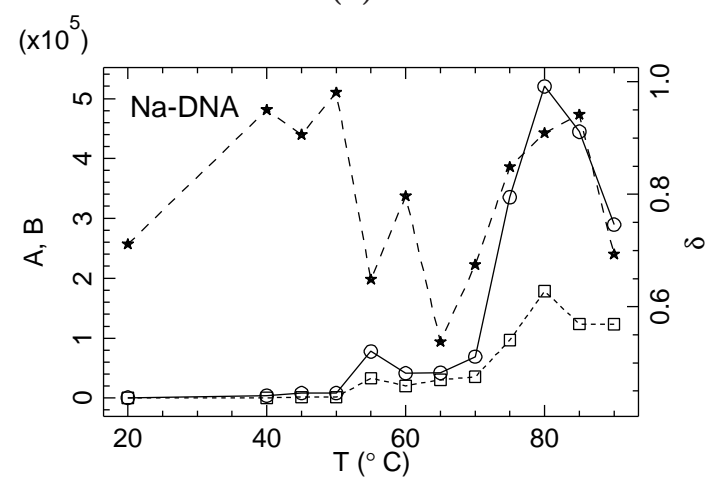

(b)

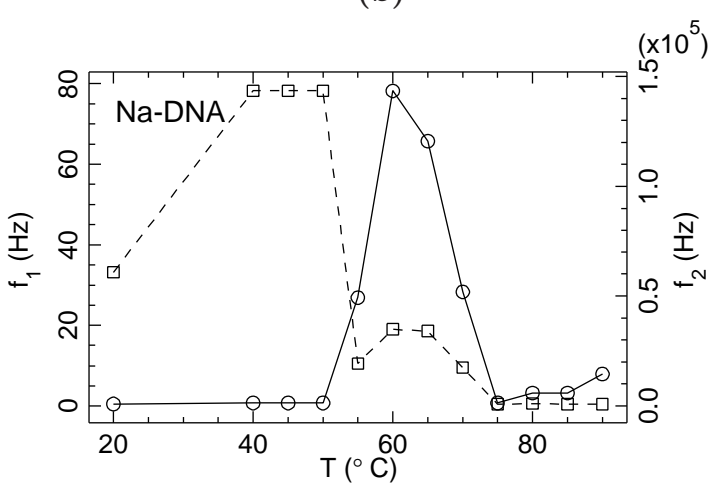

(c)

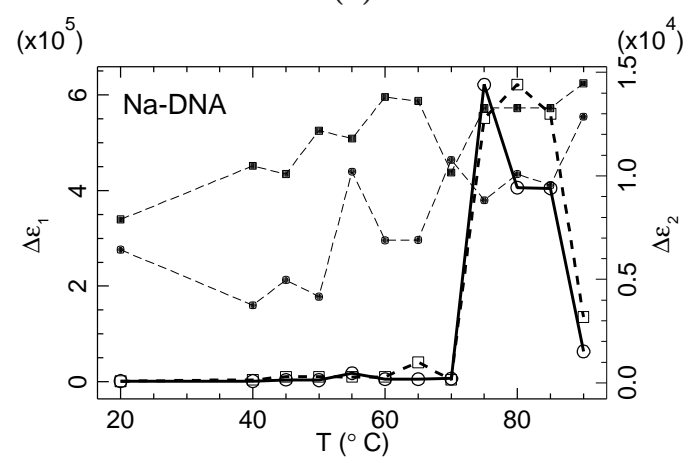

Fig. 10. Variations versus temperature of the parameters of the model fitting the dielectric response of the Na-DNA film: a) parameters $A$ (open circles), $B$ (open squares), $\delta$ (stars) of the polarization component. b) Frequencies $f_{1}$ (open circles), $f_{2}$ (open squares) of the two relaxations. c) Strengths $\Delta \epsilon_{1}$ (open circles) and $\Delta \epsilon_{2}$ (open squares) of these relaxations. The variation of the Cole-Cole exponents $\beta_{1}$ (closed circles) and $\beta_{2}$ (closed squares) are also shown on a scale ranging from 0 to 1 .

that was studied to higher temperatures than the Li-DNA film, and which has moreover a lower ionic content that drives the denaturation temperature down. The denaturation is associated to a collapse of the organized molecular structure in the film $[20,21]$. The entangled-polymer structure obtained after thermal denaturation of DNA appears to hinder the ionic mobility.
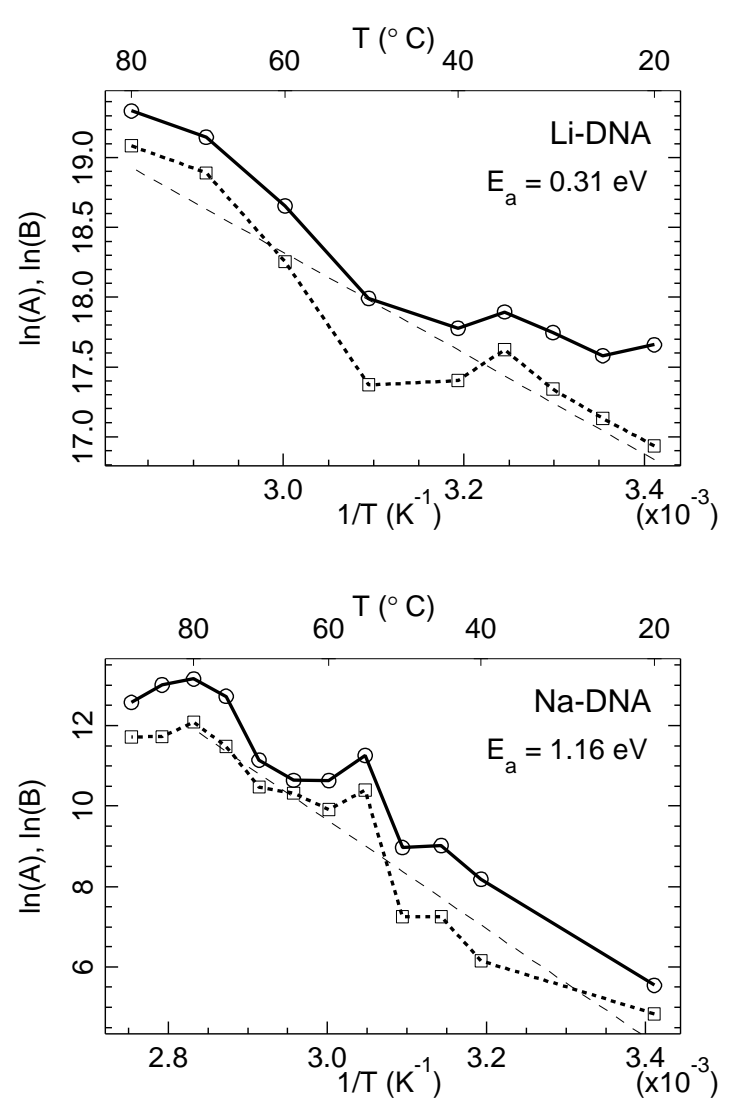

Fig. 11. Arrhenius plots of the coefficients $A$ and $B$ modeling the conductivity, i.e. plot of $\ln A$ (open circles), $\ln B$ (open squares) versus $1 / T$, where $T$ is the absolute temperature in Kelvin. Top panel: Li-DNA film, bottom panel: Na-DNA film. The oblique dash line corresponds to the linear fit of the temperature dependence of these quantities.

In addition to the thermal denaturation in the $\mathrm{Na}$ DNA film that may alter the Arrhenius behavior corresponding to the thermal activation of the ionic motions, both samples show a small peak in the $A$ and $B$ parameters followed by a decay, occurring around $35^{\circ} \mathrm{C}$ for LiDNA (see figures 6-a and 11) and $55^{\circ} \mathrm{C}$ for Na-DNA (see figures 10-a and 11). In the case of Na-DNA, which is in the A-form, one could think of a B-to-A transition due to a residual amount of B-form, that was observed in some A-DNA samples [16]. However this is unlikely because this A-to-B transition is normally observed at lower temperatures and moreover it cannot explain the similar peak in Li-DNA, which is known to be stable in the B-form, particularly at high hydration. It is more likely that this phenomenon is related to a small structural rearrangement in the film, which is associated to some changes in the ionic distribution within the sample as shown by associated changes in the two dielectric relaxations that we have observed.

The assignment of relaxations in dielectric spectroscopy is always difficult because there are many possible sources 
for polarization fluctuations. However, we have several clues that can help in this assignment.

First the frequencies of the relaxations are similar for Li-DNA and Na-DNA. The low frequency relaxation of Li-DNA is in the range $0.8 \mathrm{~Hz}$ to $20 \mathrm{~Hz}$, while it is in the range $0.45 \mathrm{~Hz}$ to $65 \mathrm{~Hz}$ for Na-DNA. For the high frequency relaxation we have $2.910^{4} \leq f_{2} \leq 1.110^{6} \mathrm{~Hz}$ for Li-DNA and $1.710^{4} \leq f_{2} \leq 1.410^{6} \mathrm{~Hz}$ for Na-DNA (except for the highest temperatures where it drops to the $\mathrm{kHz}$ range in this sample). The low values of these frequencies, and their similarity for two samples with different molecular structures, the B-form for Li-DNA and the A-form for Na-DNA, make very unlikely an assignment involving dipolar fluctuations within the DNA molecule itself.

Second, as we are working with DNA films, a rotational contribution of the molecules is excluded. The possible role of orientational fluctuations in the dielectric response of DNA has been the subject of an intense debate $[2,3,5,6$, 11]. Here the orientational fluctuations are ruled out.

This suggests that the dielectric relaxations that we observe come from the ions present in our samples, which also contribute to the measured conductivity. A recent study of low-frequency dielectric relaxations in DNA aqueous solutions [9] also found two relaxation modes, which were attributed to the role of counter-ions: a high frequency mode, in the range $0.1 \mathrm{MHz}$ to $15 \mathrm{MHz}$ is attributed to the hopping of ions between neighboring molecules and a mode at lower frequency, in the range $0.5 \mathrm{kHz}$ to $70 \mathrm{kHz}$ is connected to the persistence length of the molecules and therefore attributed to ionic motions along a single molecule. An older paper [29] also reports two modes attributed to counter-ions, a strong mode in the $1 \mathrm{~Hz}$ to $100 \mathrm{~Hz}$ range attributed to counter-ions diffusing along the molecules, and a weaker mode in the $\mathrm{Mz}$ range attributed to bound counter-ions, vibrating in the vicinity of a negative charge of the DNA strands.

It appears likely that the two relaxations that we observe have similar origins, but, as we are working with samples in which the average orientation of the molecules is known, we get further hints to confirm this assignment. As the DNA molecules lie in the plane of the film, they are almost perpendicular to the electric field used in the measurements. This should preclude the observation of the ionic diffusion along the molecules. However in our samples the molecules preserve their orientation for distances which are much longer than the $50 \mathrm{~nm}$ of the persistence length of DNA in solution. Thus we can expect lower frequencies for modes connected to ionic diffusion along the molecules. This is the case if we compare our frequencies to the $0.5 \mathrm{kHz}$ to $70 \mathrm{kHz}$ reported by [9]. Moreover the long distance diffusion could lead to very high dipolar moments. Any slight disorientation of the molecules with respect to the in-plane position can therefore lead to a sizeable response in our geometry although the field is orthogonal to the film. As a result we could nevertheless expect to detect such motions but they should show up as a weak signal only. An estimate of the relative strength of the two modes can be deduced from the magnitude of the dipole moments due to the different ionic motions. The vibrational displacement of ions bound to DNA charges or hopping between nearby molecules is of the order of a few Angströms. Displacements along the DNA molecules, in samples in which molecules are oriented, estimated to be of the order of the persistence length $(50 \mathrm{~nm})$ in [9] when the frequencies are of the order of $\mathrm{kHz}$, could rather be two orders of magnitude greater in our samples, in relation to the very low frequency of mode 1 . Therefore, if we assign the low frequency mode to motions along the DNA molecules, the corresponding dipolar moment could be at least four orders of magnitude greater than for the high frequency mode corresponding to highly localized ionic motions. Instead we find that $\Delta \epsilon_{1}$ is less than 3 orders of magnitude greater than $\Delta \epsilon_{2}$ (less than 2 orders of magnitude for Na-DNA). This is understandable in our oriented sample for experiments with an electric field which only has a very small component along the fibers. While the low frequency mode dominates the spectrum for measurements in solution [9], in our experiments with an electric field perpenducular to the film, it is hardly visible on the plots of the dielectric response function and only shows up clearly on the loss tangent figures. Therefore, taking into account all the available information, it seems reasonable to assign the low frequency relaxation to diffusion of the counter ions along the DNA molecules.

Conversely, the high frequency relaxation is strong in our measurements, which is consistent with ionic jumps between adjacent molecules, driven by the electric field transverse to the plane, as proposed in [9].

This assignment of the two relaxations that we observe explains why it is so difficult to make sense of the parameters of the relaxations, beyond the order of magnitude of their frequencies. Figures 6 and 10 show that these parameters vary widely with temperature. This is plausible because the ionic motions involved in the two relaxations are certainly highly sensitive to the local structure and ionic distribution within the samples because they are not highly local but involve longer range displacements either from a molecule to another or along a DNA molecule. Any small reorganization of the film structure due to a temperature change, a local variation of the hydration, or to the ionic displacements themselves, may affect the displacements of the ions and therefore the parameters of the relaxations. This is a difficulty of measurements with a solid sample such as our DNA films compared to solutions where the average environment of the DNA molecules is stable.

Our DNA films are similar to the wet-spun samples studied by Kutnjak et al. [12] however the emphasis of the two studies is different although they may be complementary. The question addressed in [12] is the mechanism of the electronic conductivity along the DNA molecules. This is why most of the results concern samples cut perpendicularly to the DNA fibers. Moreover the focus is on dc-conductivity, which implies the transmission of a current in the connecting wires, i.e. an electronic conductivity. However it is surprising that ref. [12] does not discuss the role of the ions which are necessarily present 
in lithium DNA. Although the ions do not contribute to the electronic dc-conductivity, at least for voltages sufficiently low to avoid ionizations or electronic recombinations at the electrodes, their presence might nevertheless affect the results discussed in [12] because, due to the electrode polarization effects they modify the distribution of the electric field inside the sample. This effect depends on the applied voltage and it might contribute to the nonlinearities discussed in [12]. Our study could therefore bring some additional elements to analyze these experiments. The paper also presents some results on the frequency dependence of the conductivity. The general trend is a power law increase similar to the one that we show in Fig. 4 but the data are not accurate enough to allow a quantitative comparison. They nevertheless raise a question because we show that effects that we can unambiguously attribute to mobile ions by comparing different ionicities appear similar to results attributed to hopping hole conduction in [12]. Further investigations to clarify this point, with different sample geometries or ionicity, would be interesting.

\section{Conclusion}

As in earlier dielectric studies of DNA in solution, the measurements on films made of DNA fibers meets the difficulty of the strong conductivity of the samples, which leads to a very strong electrode polarization response that tends to dominate the dielectric relaxations, but gives however some information on the mobility and diffusivity of the charge carriers. There are nevertheless various interests in studying DNA in this fiber form:

- By avoiding the rotations of the molecules, it eliminates one possible contribution to the dielectric fluctuations,

- The orientation of the molecules in the films can moreover be used for the assignment of the dielectric relaxations,

- and perhaps more importantly this allowed the first dielectric study of DNA in its A-form, with a molecular structure which is significantly different from that of the familiar B-DNA. It turns out however, that, as the low-frequency dielectric response is due to the dynamics of the counter-ions, which contribute to the stabilization of the DNA structure, the two forms show qualitatively similar dielectric behaviors. However the activation energy for the ionic motions is significantly higher in A-DNA fibers, which are more crystalline and less hydrated, leaving less room for the ions to move. It would be interesting to study similar film samples in the radio-frequency domain, allowing the observation of polarization effects coming from the internal structure of the DNA molecule itself.

We would like to thank A. Wildes (ILL Grenoble) and J. Richard (Institut Néel, Grenoble) for useful discussions and Alain Sylvestre (G2ELab Grenoble) for supporting the dielectric measurements. We also would like to thank Emmanuel
André from Pôle de Capteurs Thermométriques et Calorimétrie of the Institut Néel for his support in the realization of the optical microscopy observations.

\section{References}

1. G. Jungner, I. Jungner and L.-G. Allgén, Nature 163, 849 (1949)

2. S. Takashima, J. Mol. Biol. 7455 (1963)

3. S. Takashima. J. Phys. Chem. 70, 1372 (1966)

4. M. Hanss, Bioplymers 4, 1035 (1966)

5. M. Sakamoto, H. Kanda, R. Hayakawa, and Y. Wada. Biochemica 15, 879 (1976)

6. M. Sakamoto, R. Hayakawa and Y. Wada, Biopolymers 17, 1507 (1978)

7. M. Sakamoto, R. Hayakawa, and Y. Wada. Biopolymers $182769(1979)$

8. M. S. Tung, R. J. Molinari, R. H. Cole, and J. H. Gibbs. Biopolymers 162653 (1977)

9. S. Tomić. S. Dolanski Babić, T. Vuletić, S. Krea, D. Ivanković, L. Griparić and R. Podgornik, Phys. Rev. E 75, 021905 (2007)

10. M. Mandel, Ann. N.Y. Acad. Sci. 303, 74-89 (1977)

11. R.H. Cole, Ann. N.Y. Acad. Sci. 303, 59 (1977)

12. Z. Kutnjak, C. Filipič, R. Podgornik, L. Nordenskiöld and N. Korolev, Phys. Rev. Lett. 90; 098101 (2003)

13. W. Saenger, Principles of Nucleic Acid Structure, Springer Verlag, Berlin (1984)

14. J.D. Watson and F.H.C. Crick, Nature 171737 (1953)

15. W. Fuller, Nature 424, 876 (2003)

16. J. Valle-Orero, A. Wildes, J-L Garden, and M. Peyrard. J. Phys. Chem. B 1171849 (2013)

17. S.M. Lindsay, S.A. Lee, J.W. Powell, T. Weidlich, C. Demarco, G.D. Lewen, J.N. Tao and A. Rupprecht, Biopolymers 27 1015-1043 (1988)

18. A. Rupprecht, Acta Chemica Scandinavica 20494 (1966)

19. A. Rupprecht. Biochimica et Biophysica Acta 199277 (1970)

20. A.Wildes, Nikos Theodorakopoulos, Jessica Valle-Orero, Santiago Cuesta-López, Jean-Luc Garden, and Michel Peyrard, Phys. Rev. E 83, (2011) 061923

21. J. Valle-Orero, J-L Garden, J. Richard, A. Wildes, and M. Peyrard. J. Phys. Chem. B 1164394 (2012)

22. S. Sorriso and A. Surowiec, Advances in Molecular Relaxation and Interaction Processes 22, 259 (1982)

23. P. Lunkenheimer, V. Bobnar, A.V. Pronion, A.I. Ritus, A.A. Volkov, and A. Loidl, Phys. Rev. B 66 052105-1-4 (2002)

24. A.K. Jonscher, Nature 267 673-678 (1977)

25. K.S. Cole and R.H. Cole, J. Chem. Phys. 9 (1941) 341.

26. S. Havriliak, S. Negami, Polymers 8, (1967) 161-210

27. Paul Ben Ishai, Mark S Talary, Andreas Caduff, Evgeniya Levyand and Yuri Feldman, Meas. Sci. Technol. 24102001 (2013)

28. A.A. Kornyshev and M.A. Vorotyntsev, Electrochim. Acta 26 303-323 (1981)

29. J. Baker-Jarvis, C.A. Jones and B. Riddle, NIST Technical note 1509, 1 (1988) 